\title{
Correction to: Fixation of polyvinylidene fluoride (PVDF) mesh with cyanoacrylate-derived glues in a rat experimental model: histopathologic immunohistochemical and morphometric study
}

\author{
A. Poli ${ }^{1}$ (I) $\cdot$ F. Parisi ${ }^{1} \cdot$ F. Millanta $^{1} \cdot$ L. Solfanelli ${ }^{2} \cdot$ P. García-Pastor $^{3} \cdot$ C. Magliaro $^{4} \cdot$ V. Miragliotta $^{1} \cdot$ S. Burchielli $^{5}$
}

Published online: 5 February 2020

(c) Springer-Verlag France SAS, part of Springer Nature 2020

\section{Correction to: Hernia \\ https://doi.org/10.1007/s10029-019-02078-5}

Unfortunately, during the revision of the manuscript the title has been incorrectly published

The correct title has been copied below.

"Fixation of polyvinylidene fluoride (PVDF) mesh with cyanoacrylate-derived glues in a rat experimental model: histopathologic immunohistochemical and morphometric study"

The original article has been corrected.

The original article can be found online at https://doi.org/10.1007/ s10029-019-02078-5.

\section{A. Poli}

alessandro.poli@unipi.it

1 Dipartimento Di Scienze Veterinarie, Università Di Pisa, Viale delle Piagge, 2, 56124 Pisa, Italy

2 Dipartimento Di Medicina Clinica E Sperimentale, Università Di Pisa, Pisa, Italy

3 Abdominal Wall Surgery Unit, Surgery Department, La Fe University and Politechnic Hospital, Valencia, Spain

4 Centro Di Ricerca “E. Piaggio”, Università Di Pisa, Pisa, Italy

5 Fondazione Toscana "Gabriele Monasterio" E Centro Di Biomedicina Sperimentale del CNR, Pisa, Italy
Publisher's Note Springer Nature remains neutral with regard to jurisdictional claims in published maps and institutional affiliations. 